\title{
Heart
}

\section{AT THE HEART OF THE CONFLICT}

\begin{tabular}{|c|c|}
\hline Journal: & Heart \\
\hline Manuscript ID & heartjnl-2019-315010.R1 \\
\hline Article Type: & Editorial \\
\hline $\begin{array}{r}\text { Date Submitted by the } \\
\text { Author: }\end{array}$ & $n / a$ \\
\hline Complete List of Authors: & $\begin{array}{l}\text { Ansbro, Eimhin; London School of Hygiene and Tropical Medicine Faculty } \\
\text { of Public Health and Policy } \\
\text { Perel, Pablo; London School of Hygiene and Tropical Medicine Faculty of } \\
\text { Epidemiology and Population Health, Centre for Global Chronic } \\
\text { Conditions }\end{array}$ \\
\hline Keywords: & $\begin{array}{l}\text { Coronary artery disease }<\text { DISEASES, Cardiac risk factors and } \\
\text { prevention < DISEASES, Health Services < RESEARCH APPROACHES, } \\
\text { Global health < RESEARCH APPROACHES }\end{array}$ \\
\hline Abstract: & $\begin{array}{l}\text { Cardiovascular disease (CVD) prevalence and mortality are rapidly } \\
\text { increasing globally and this burden, especially in premature CVD death, } \\
\text { is disproportionally felt in low-and middle-income countries (LMIC)1. We } \\
\text { know, in addition, that most current armed conflicts and resultant } \\
\text { population displacement occur in LMICs } 2 \text {. There is also evidence that } \\
\text { factors associated with increased CVD risk, such as tobacco and alcohol } \\
\text { use, are heightened as people cope with conflict and the post-conflict } \\
\text { environment } 1,3 \text {. Recent reviews have shown that intense emotion, } \\
\text { especially bereavement, may trigger heart attacks, but most of these } \\
\text { studies have been conducted in stable settings } 4 \text {. So, although it may } \\
\text { seem logical that CVD morbidity and mortality would increase in armed } \\
\text { conflict settings, it is surprising that there is such limited evidence to } \\
\text { date in this area. }\end{array}$ \\
\hline
\end{tabular}

\section{SCHOLARONE Manuscripts}


Title: At The Heart Of The Conflict

Authors: Éimhín Ansbro ${ }^{1}$, Pablo Perel ${ }^{1}$

${ }^{1}$ Centre for Global Chronic Conditions, London School of Hygiene and Tropical Medicine, London, United Kingdom.

\section{Corresponding author:}

Name: Pablo Perel,

Address: London School of Hygiene and Tropical Medicine, Keppel St, Bloomsbury, London WC1E 7HT, United Kingdom.

E-mail: pablo.perel@Ishtm.ac.uk

Telephone: +44(0) 2079588120

Fax: +44 (0) 2079272701

Keywords: cardiovascular diseases; diagnostic technique, cardiovascular; relief work: armed conflicts; humanitarian

Word Count: 1500 
Cardiovascular disease (CVD) prevalence and mortality are rapidly increasing globally and the burden, especially of premature CVD death, is disproportionally felt in low-and middleincome countries (LMIC) ${ }^{1}$. We know, in addition, that most current armed conflicts and resultant population displacement occur in LMICs ${ }^{2}$. There is also evidence that factors associated with increased CVD risk, such as tobacco and alcohol use, are heightened as people cope with conflict and the post-conflict environment 1,3 . Recent reviews have shown that intense emotion, especially bereavement, may trigger heart attacks, but most of these studies have been conducted in stable settings ${ }^{4}$. So, although it may seem logical that CVD morbidity and mortality would increase in armed conflict settings, it is surprising that there is such limited evidence to date in this area.

This first published systematic review to examine current evidence on the association between armed conflict and cardiovascular disease risk by Jawad et al. is both timely and important ${ }^{5}$. The authors sought to address the question: "What is the association between armed conflict and CVD risk for civilians in LMICs, compared to civilians with less or no exposure to armed conflict?" They reported on 65 quantitative studies (covering 23 armed conflicts), which included a comparator group or examined outcomes over time. The authors descriptively summarised the studies using harvest diagrams, grouping by risk factor, setting and date of publication. They found evidence that armed conflict is associated with overt illness, including increased coronary heart disease, cerebrovascular, and endocrine diseases; and with an increase in CVD risk factors: blood pressure, lipids, alcohol, and tobacco use. In addition, the added risk may become evident both during periods of active conflict and in the acute and chronic post-conflict period.

The authors succeeded in clearly summarising the existing research. The main limitations of this review relate to the quality of the evidence itself. Among the papers meeting their inclusion criteria, we highlight the following limitations: basic study design (almost half were cross-sectional), low quality (only $10 \%$ were considered high quality), poor measurement of outcomes (for example, over half used self-report or didn't specify how outcomes were measured), lack of exploration of causal pathways (potentially useful to design preventive interventions), and lack of studies including refugees or relevant regions like the Middle East (approximately half of the included studies were derived from just three conflict settings in the Balkans and Colombia). As pointed out by the authors, the important inconsistency in their findings with respect to different outcomes with similar causal pathways (an increase in mortality from chronic ischaemic heart disease but no change in the effect on myocardial infarction, angina pectoris or chronic ischaemic chronic disease) may reflect methodological shortcomings of the included studies.

The authors make sensible recommendations for conducting future, more robust research, which will address some of the limitations of the current evidence base. They suggest we focus on: using better outcome measurement, improving management of confounding, and increasing the use of control arms or quasi-experimental study designs such as interrupted time series.

This review and a recent review of diabetes care in humanitarian crises both highlight the impact of the increasing global burden of noncommunicable diseases (NCDs) on crisis settings and support advocacy work to bring NCDs, including CVD and the excess crisisassociated risks, squarely onto the agendas of humanitarian and development agencies, funders and the research community ${ }^{6}$. In addition to better characterising the impact of conflict on CVD risk, it is also vital to conduct high quality research to improve the clinical care and programmatic response for people with CVD in conflict settings. Although the evidence base is growing, there has been limited evidence on effective interventions or guidance for CVD (and NCDs in general) in humanitarian settings ${ }^{7}$. For example, the WHO PEN guide is limited in scope and humanitarian actors, such as MSF, acknowledge that their current guidelines could be adapted and simplified ${ }^{8,9}$.

A research roadmap could be designed around the response of humanitarian organisations to CVD (and other NCDs) in crisis settings. Among the main research priorities we highlight:

1) Rapid assessment tools: We do not know the pre-conflict burden of CVD in most crisis 
contexts. Therefore, some simple and reliable rapid assessment tools could be valuable for informing humanitarian organisations operations in specific settings. Guidance for NCD care providers on adapting the response by stage and character of humanitarian crisis (acute emergency, stable or unstable protracted conflict or post-conflict) and by the underlying state and resilience of the health system in the conflict-affected area (fragile vs. more mature health system) may also be useful.

2) Simple diagnostic tools and approaches: The diagnosis of CVD requires investigations involving equipment, personnel and training - that are not widely available, accessible or affordable in low-income settings with fragile health systems. So, we need to develop and test feasible, accurate and context-adapted point-of-care diagnostic tools and approaches. Such approaches may assist, for example, in distinguishing CVD-induced chest pain or shortness of breath from sputum negative TB at primary care level in countries with a high TB burden.

3) Individual risk stratification: People with established CVD, or those at high risk, are particularly susceptible to exacerbations or acute events triggered by stress or treatment interruption. A more solid, evidence-based risk stratifying approach could help to target interventions according to risk, for example, prioritising high-risk groups and urgent interventions during the emergency phase of a crisis. This may mean simply investing more in identifying people with diabetes and chronic kidney disease, both groups with elevated CVD risk.

4) Appropriate models of care: We echo the authors' call for the post-conflict (or inter-conflict) reconstruction phase to include low resource, primary level preventive interventions for CVD. Identifying and achieving good blood pressure and glycaemic control among people with hypertension and diabetes, taking a CVD risk management approach and providing good quality CVD secondary prevention care warrant a clear focus. Ideally, NCD interventions in general should be designed using a health systems-based approach, with a person-centred care focus, while applying the learning from humanitarian organisations' decades of work on chronic HIV care. Empowering nurses and other non-medical health worker cadres to diagnose and manage stable patients, empowering patients to self-care, decentralising aspects of care to community level (e.g. monitoring, dispensing or delivery of medications), and utilising fixed dose combination drugs for hypertension and CVD secondary prevention may all play a part in such interventions. Importantly, humanitarian interventions that target CVD should be integrated with other chronic conditions, such as diabetes, asthma and HIV; integrated into pre-existing primary care where possible; and integrated across health system levels and sites, which are linked by robust referral pathways. Additionally, it would seem sensible to integrate mental health and psychosocial support with other aspects of care to address some of the stress-related CVD risk associated with conflict. Although the suggested approaches for models of care listed above look promising, we need to conduct robust implementation research (using mixed methods) to better understand what works, for whom, and in which crisis contexts.

5) Ensuring availability and affordability of essential medicines and technologies: An NCD model of care will only work if essential medicines and technologies are available, accessible and affordable. Supply and access are often limited at baseline and worsen in an emergency, when supply chains may be disrupted. In working to address this, from 2017, the Interagency Emergency Health Kit (IEHK), designed to provide for the primary care needs of 10,000 people for approximately three months in post-emergency settings, has included medications to treat acute NCD exacerbations. In addition, a specific modular NCD emergency health kit is currently being piloted in Iraq and Syria ${ }^{10}$. Further research is needed to document the effectiveness of this strategy and to explore other financing and procurement approaches to ensure availability and affordability in the longer term.

There are some crosscutting issues to be considered across all the proposed research areas. Firstly, collaboration of key stakeholders is vital. These include: humanitarian and development actors, academics, international agencies, governments, people affected by conflicts and the private sector. Secondly, conducting research in conflict settings involves ethical and logistical challenges, so we need to develop innovative methodological approaches to conduct robust research in these settings. Finally, there is a need for more 
economic evaluations to ensure the efficiency of responses to NCD needs in humanitarian crises.

To conclude, while calling for improved research in this area, we acknowledge the significant work already underway by humanitarian actors and UN bodies to address the burden of NCDs in crisis settings. We suggest focussing on what works, both by promoting the delivery of proven, cost effective CVD interventions and by supporting governments to address CVD risk factors through community level prevention strategies and through alcohol, tobacco and food policy strengthening. The aim is to minimise the impact of distress and disruption on people with CVD in crises and to build better systems to keep hearts healthy into the future.

We thank Dr Helen Bygrave, Médecins sans Frontières, for her review and helpful comments and humanitarian NGO colleagues with whom we collaborate.

The Corresponding Author has the right to grant on behalf of all authors and does grant on behalf of all authors, an exclusive licence (or non exclusive for government employees) on a worldwide basis to the BMJ Publishing Group Ltd and its Licensees to permit this article (if accepted) to be published in HEART editions and any other BMJPGL products to exploit all subsidiary rights.

1. Global Burden of Disease Study 2013 Collaborators T, Barber RM, Bell B, et al. Global, regional, and national incidence, prevalence, and years lived with disability for 301 acute and chronic diseases and injuries in 188 countries, 1990-2013: a systematic analysis for the Global Burden of Disease Study 2013. Lancet (London, England). 2015;386(9995):743-800. doi:10.1016/S0140-6736(15)60692-4

2. UNHCR. Global Trends: Forced Displacement in 2015. Geneva; 2016. https://s3.amazonaws.com/unhcrsharedmedia/2016/2016-06-20-global-trends/201606-14-Global-Trends-2015.pdf. Accessed June 25, 2016.

3. Roberts B, Patel P, McKee M. Noncommunicable diseases and post-conflict countries. Bull World Health Organ. 2011;90(1):2-2A. doi:10.2471/BLT.11.098863

4. Nawrot TS, Perez L, Künzli N, Munters E, Nemery B. Public health importance of triggers of myocardial infarction: a comparative risk assessment. Lancet. 2011;377(9767):732-740. doi:10.1016/S0140-6736(10)62296-9

5. Jawad, Mohammed; Vamos, Eszter P.; Najim, Muhammad; Roberts, Bayard; Millett C. The impact of armed conflict on cardiovascular disease risk: a systematic review. Heart. 2019.

6. Kehlenbrink S, Smith J, Ansbro É, et al. The burden of diabetes and use of diabetes care in humanitarian crises in low-income and middle-income countries. Lancet Diabetes Endocrinol. 2019;0(0). doi:10.1016/S2213-8587(19)30082-8

7. Ruby A, Knight A, Perel P, Blanchet K, Roberts B. The Effectiveness of Interventions for Non-Communicable Diseases in Humanitarian Crises: A Systematic Review. Shimosawa T, ed. PLoS One. 2015;10(9):e0138303. doi:10.1371/journal.pone.0138303

8. WHO | Package of essential NCD interventions for primary health care: cancer, diabetes, heart disease and stroke, chronic respiratory disease. WHO. 2014.

9. Ansbro E. Mixed Methods Evaluation of MSF Primary Care Based NCD Service in Irbid, Jordan (Feb 2017- Feb 2018).; 2018. http://fieldresearch.msf.org/msf/handle/10144/619309.

10. Slama S, Lee J, Aragno M, Laroche S, Hogerzeil H. The development of the noncommunicable diseases emergency health kit. East Mediterr Heal J. 2018;24(01):92-98. doi:10.26719/2018.24.1.92 